\title{
JAK-STAT signaling in asthma
}

\author{
Alessandra B. Pernis ${ }^{1}$ and Paul B. Rothman ${ }^{2}$ \\ ${ }^{1}$ Department of Medicine, and \\ ${ }^{2}$ Department of Microbiology, Columbia University, New York, New York, USA \\ Address correspondence to: Alessandra Pernis, Department of Medicine, \\ Columbia University, 630 West 168th Street, New York, New York 10032, USA. \\ Phone: (212) 305-3763; Fax: (212) 305-4478; E-mail: abp1@columbia.edu.
}

J. Clin. Invest. 109:1279-1283 (2002). doi:10.1172/JCI200215786.

The past two decades have witnessed a dramatic increase in the prevalence of asthma worldwide (1). Asthma is a chronic disease characterized by variable airway obstruction, airway hyperresponsiveness (AHR), and airway inflammation and remodeling. Histological studies show that airways of asthmatic patients contain a chronic inflammatory infiltrate composed of lymphocytes, eosinophils, and mast cells. This infiltrate is usually accompanied by desquamation of the bronchial epithelial layer, goblet cell hyperplasia, and thickening of the submucosa. In most cases, the asthmatic inflammatory process results from inappropriate immune responses to common environmental antigens in a genetically susceptible individual (2). These inappropriate immune responses are orchestrated by a subset of $\mathrm{CD} 4^{+} \mathrm{T}$ helper cells termed $\mathrm{T}$ helper 2 (Th2) cells.

Cytokines play a pivotal role in the development of asthma by regulating the expansion of Th 2 cells and by mediating many of the Th2 effector functions that underlie the pathogenic events of an asthmatic response. Much effort has recently been placed in elucidating the pathways used by cytokines to mediate their actions. These studies have revealed that cytokine-mediated signals are primarily transduced by the Jak-Stat signaling cascade (3). In this review we will highlight the recent advances made in dissecting the roles of this signaling pathway in the pathogenesis of asthma.

\section{Jak-Stat signaling in Th1 and Th2 differentiation}

The two major subsets of $\mathrm{CD}^{+}{ }^{+}$Th cells, termed Th 1 and Th2, secrete mutually distinct profiles of cytokines and thereby coordinate different classes of immune response (4). Th 1 cells secrete IL-2, IFN- $\gamma$, and TNF- $\beta$, whereas Th 2 cells produce IL- 4 , IL-5, IL-6, IL-10, and IL-13. It is now well accepted that Th1 cells are critically involved in the generation of delayed-type hypersensitivity responses, whereas Th2 cells can direct $B$ cells to mount strong humoral responses. Polarization of an immune response toward a Th2 phenotype, while extremely useful in the clearance of parasites, may prove harmful if directed against an otherwise innocuous environmental antigen, as occurs in the pathogenesis of allergic diseases like asthma.

The Th2 cytokines, primarily IL-4, IL-5, and IL-13, control all the major components that characterize an inflammatory asthmatic response, including IgE isotype switching, mucus production, and the recruitment and activation of eosinophils. The involvement of Th2 cells in the pathophysiology of asthma has been corroborated by studies in both humans and mice. The population of Th2 cells is notably expanded in the airways of asthmatic subjects, and presence of these cells correlates with AHR and airway eosinophilia (2). Work in murine models of AHR confirms this correlation and demonstrates that adoptively transferred antigenspecific Th2, but not Th1, cells can mediate airway eosinophilia, mucus hypersecretion, and AHR when recipient mice are exposed to inhaled antigen (5).

The cytokines IL-12 and IL-4 direct the differentiation of Th1 and Th2 cells, respectively, from naive $T$ helper cells $(6,7)$. Genetic studies have confirmed the physiologic importance of these cytokines in vivo. Mice deficient in either IL-12 or the IL-12 receptor (IL-12R) are unable to mount Th1 responses, while mice lacking IL-4 or the IL-4 receptor $\alpha$ (IL-4R $\alpha$ ) chain display defects in the generation of Th2 responses $(8,9)$. Indeed, IL-12 and IL-4 not only drive the expansion of their corresponding Th subset but simultaneously block the generation of the opposing subset. Given the importance of IL-4 and IL-12 in controlling Th differentiation, much effort has been placed over the past few years in dissecting the mechanisms by which these cytokines mediate their actions. These studies have revealed that, like most other cytokines, IL-4 and IL-12 activate the Jak-Stat signaling cascade discussed elsewhere in this Perspective series. In this signaling pathway, binding of a cytokine to its receptor leads to the activation of members of the JAK family of receptorassociated kinases. These kinases subsequently activate, via tyrosine phosphorylation, preexistent cytoplasmic factors termed Stats (signal transducer and activator of transcription). Tyrosine phosphorylation allows the 


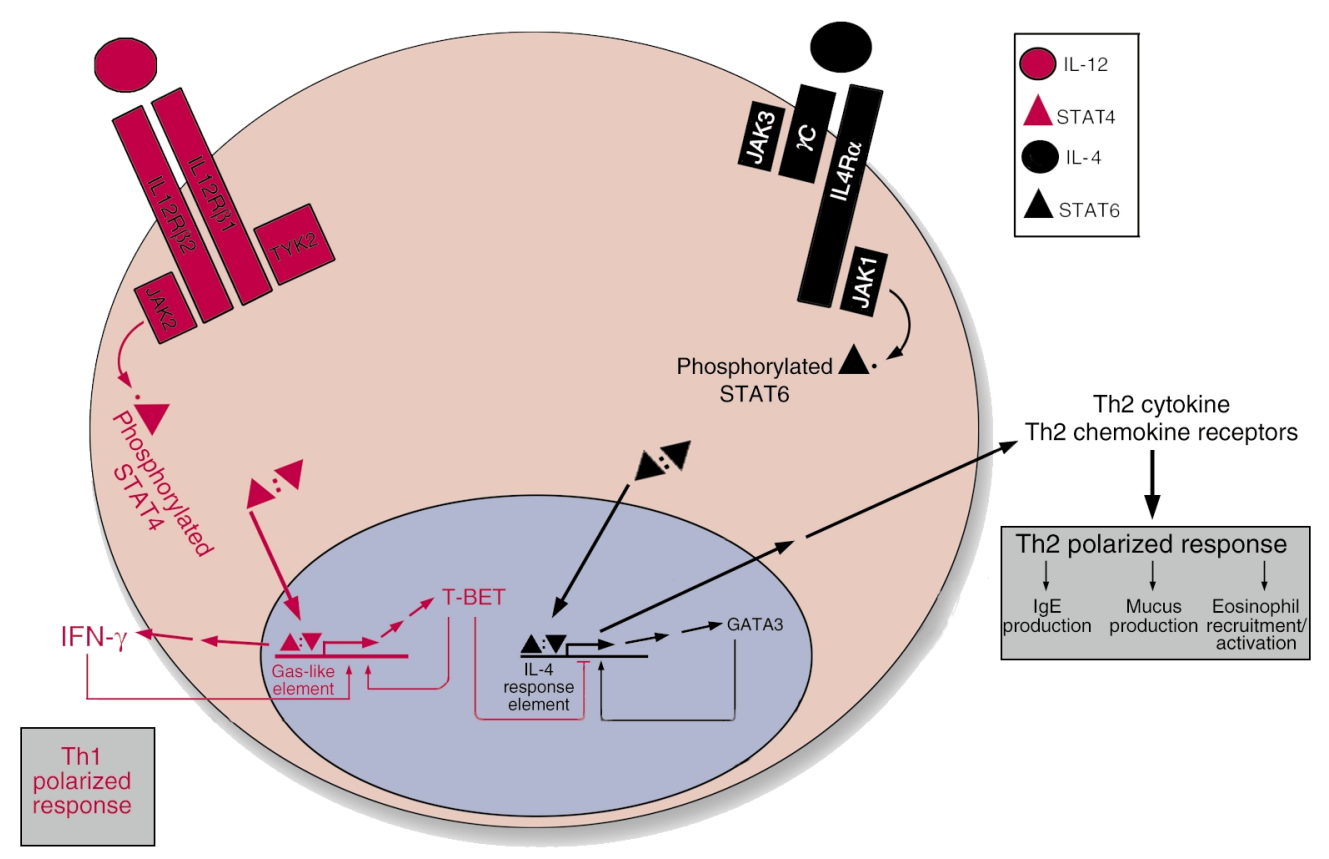

\section{Figure 1}

JAK-STAT signaling and the generation of Th1 and Th2 cells. Following antigen presentation, a naive CD4+ T cell will differentiate along the Th1 or Th2 pathway, depending on the nature of the cytokines it contacts. Signaling through the IL-12 receptor (red pathway) and its associated Jak and Stat proteins culminates in the expression of Th1-specific gene products, particularly the cytokine interferon- $\gamma$ (IFN- $\gamma$ ) Conversely, IL- 4 activates a distinct receptor complex, containing different Jaks and Stats, and favors the expression of Th2 cytokines and other gene products (black pathway). These pathways can regulate one another at the transcriptional level. In particular, the transcription factor GATA3, whose expression is induced by IL4 signaling, is essential for the transactivation of other Th2-associated genes, such as those for Th2 cytokines and chemokine receptors. Another transcription factor, T-BET, is induced by IFN- $\gamma$ (and therefore indirectly by IL-12 stimulation) and also favors expression of this cytokine - thus establishing a positive feedback loop that supports Th1 polarization. In addition, T-BET silences expression of Th2-associated genes. Polarization toward the Th2 response leads to the expression of various cytokines - IL-4, IL-5, IL-6, IL-10, and IL-13 - that contribute to the pathologies seen in asthma.

Stat proteins to dimerize and translocate to the nucleus, where they mediate changes in gene expression by binding specific DNA elements.

Although both IL-4 and IL-12 follow this basic signaling framework, the two cytokines differ in the specific Jak and Stat components that they activate (10). IL-4 stimulates Jak1 and Jak3 to activate Stat6. In contrast, interaction of IL- 12 with its receptor leads to the activation of Jak 2 and Tyk2 and the subsequent phosphorylation of Stat 4. Activation of Stat 6 and Stat 4 are thus critical events in the signaling cascades of IL- 4 and IL-12, respectively. Given the pivotal roles of these two cytokines in skewing Th cells toward either a Th2 or a Th1 phenotype, it is not surprising that Stat 6 and Stat 4 control multiple aspects of the Th differentiation programs, as shown in Figure 1 and detailed below.

\section{Stat6 in Th differentiation}

The importance of Stat 6 in Th 2 cell differentiation has been confirmed by the generation of Stat6-deficient mice (11-13), which fail to mount Th2 responses either in vitro - in Th differentiation systems - or in vivo, upon infection with parasitic pathogens that elicit Th2 responses. Th2 memory cell development and survival are dependent on Stat6 $(14,15)$, whereas Stat6-independent pathways of IL-4 production seem to contribute only to the initial responses mounted by naive
$\mathrm{T}$ cells, rather than to the later regulation of the Th2 population. Stat 6 appears to be not only necessary but also sufficient to drive Th2 differentiation, since introduction of constitutively active forms of Stat 6 results in the expression of Th2-type cytokines even when this form of Stat 6 is expressed in cells already differentiating toward a Th1 phenotype $(16,17)$.

Stat6's role in Th2 differentiation is not confined to activating and silencing the expression of specific cytokines but extends to other aspects of the distinctive pattern of gene expression found in Th2 cells, including the heightened expression in these cells of the chemokine receptors CCR4 and CCR8 (18). Stat6deficient $T$ cells are significantly impaired in their ability to expand upon IL-4 stimulation, thus implicating this transcription factor in IL-4-driven proliferation as well as differentiation $(17,19)$. This defect is due to a block in the progression from the G1 to the $S$ phase of the cell cycle and correlates with an impaired ability of Stat6-deficient T cells to downregulate the cell cycle-dependent kinase inhibitor $\mathrm{p} 27^{\mathrm{kip}}$ in response to IL-4 stimulation. In parallel with its induction of Th2specific gene expression programs, Stat 6 appears to suppress Th1-specific pathways. Indeed, generation of mice deficient in both Stat 4 and Stat 6 has revealed that in the absence of Stat6, Stat4-deficient $\mathrm{CD}_{4}^{+} \mathrm{T}$ cells can differentiate into Th1 cells (20). This effect is 
not detected in mice deficient for Stat 4 alone, suggesting that the presence of Stat 6 blocks the ability of Th cells to acquire the Th1 phenotype.

The mechanisms by which Stat 6 controls Th2 differentiation are complex and involve subtype-specific induction of specific transcription factors, as well as changes in the chromatin structure and the pattern of cytosine methylation at the IL4 locus. Th2specific factors like GATA3 and c-maf synergize with NFAT and AP-1, which are more broadly expressed $(6,7)$, to activate the characteristic Th2 pattern of gene expression. While both the transcriptional and the epigenetic changes depend on Stat6, it remains unclear whether either of these effects is direct. Some reports also suggest that Stat 6 can bind to specific sites within the IL4 promoter and to a site in the $3^{\prime}$ untranslated region of IL4 that may function as a Th1-specific silencer (21-23). The significance of Stat 6 targeting to these regulatory regions for Th2 commitment has not been established.

\section{Stat4 in Th differentiation}

While activation of Stat 6 in response to IL-4 is critical for the generation of Th2 responses, activation of a different Stat, Stat 4 , skews Th cells toward the Th1 phenotype following IL-12 stimulation. Phenotypic analysis of Stat4-deficient mice shows that activation of Stat 4 is critical to this process. Stat4-deficient $T$ cells are unable to produce high levels of the Th1 cytokine IFN- $\gamma$ after exposure to IL-12 $(20,24)$. Stat4-independent pathways of Th1 differentiation also exist, but, as mentioned above, their effects are seen most clearly in the absence of Stat6 $(20,24)$. Recent studies suggest that Stat 4 activation is particularly critical for the sustained, rather than the initial, production of Th1-type cytokines (15). Stat4 also mediates the downregulation of the cell-cycle inhibitor $\mathrm{p} 27^{\mathrm{kip}}$ in IL-12-treated Th1 cells $(19,25)$, allowing it (like Stat6) to control Th proliferation as well as differentiation.

As with Th2 cytokines, induction of Th1-specific cytokine gene expression requires epigenetic remodeling, as well as the induction of Th1-specific transcription factors like the recently described T-bet (26). The precise mechanisms employed by Stat 4 to mediate its effects on Th1 differentiation have not been fully delineated. In particular, it is unclear whether Stat 4 activation participates in the induction of the Th1-specific factor T-bet, whose deficiency in mice has recently been shown to lead to the spontaneous development of airway changes characteristic of human asthma $(15,25$, $27,28)$. Stat 4 is reported to transactivate IFN- $\gamma$ directly (29), although additional factors are likely to be required for full expression of this cytokine (7). Activated Stat 4 also blocks differentiation along the Th2 pathway by repressing expression of the Th2-specific factor Gata3 (30), establishing yet another parallel between the role of Stat 4 in Th1 development and that of Stat 6 in Th2 development. Further progress toward understanding the physiological basis of the actions of these two Stat proteins will require the identification of the key targets of these two transcription factors.

\section{The Jak-Stat signaling cascade in IgE regulation}

Levels of IgE correlate with the incidence of atopic asthma in humans. The recent success of anti-IgE therapy in the treatment of atopic asthmatics also highlights the importance of this class of antibodies in human asthma (31) (Figure 2). Work over the past ten years has

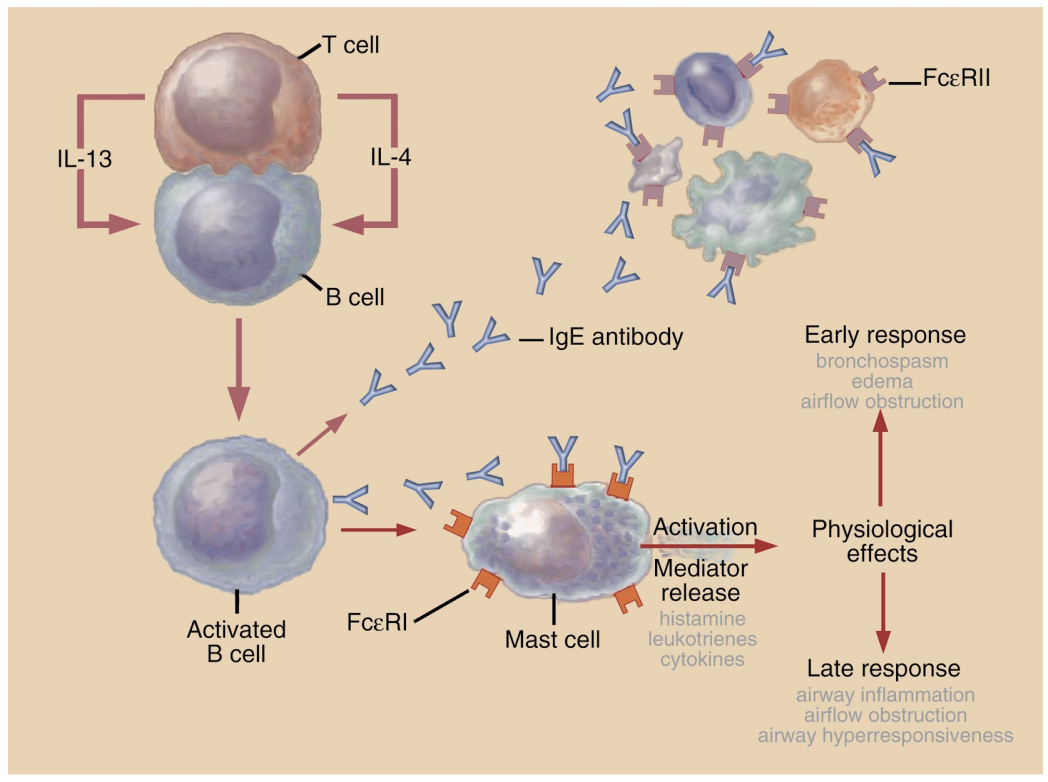

\section{Figure 2}

IgE in the pathogenesis of asthmatic responses. Production of Th2-type cytokines (IL-4 and IL-13) by T cells in response to antigens like airborne allergens will drive IgE synthesis by B cells. IgE can then bind to high-affinity IgE receptors on mast cells. Cross-linking of the bound IgE molecules upon reexposure to the antigen provokes mast cell degranulation with the subsequent release of a variety of mediators, which trigger both early and late inflammatory asthmatic responses. 
detailed how cytokines (IL-4 and IL-13, in particular) induce the production of IgE and how other cytokines, such as IFN- $\gamma$, block this induction. IL-4 initiates signaling by oligomerizing the heterodimeric IL-4 receptor, which, in hematopoietic cells, is composed of the ligand-specific IL-4R $\alpha$ chain and the common $\gamma$ chain $(\gamma \mathrm{C})(32)$. This oligomerization initiates signaling by activating Jak 1 and Jak3, which associate constitutively with the cytoplasmic tail of cytokine receptor subunits (Jak1 with IL-4R $\alpha$ and Jak3 with $\gamma$ C). IL-13 likewise binds IL-4R $\alpha$ and can activate signaling through Jak1, but this cytokine also binds a more specific receptor subunit, the IL-13R $\alpha 1$ chain, which associates with the Jak-family kinase Tyk2.

After binding of either IL-4 or IL-13, the activated Jaks phosphorylate tyrosines within the cytoplasmic domain of the IL-4R $\alpha$, which act as docking sites for Stat6. Phosphorylated Stat6 homodimerizes, translocates to the nucleus, and activates transcription of genes involved in B cell differentiation, including the germline $I \varepsilon$ and $I \gamma 1$ genes in mice and germline $I \varepsilon$ and I $\gamma 4$ genes in humans (33). Induction of these germline promoters, and expression of the corresponding germline "sterile" transcripts, have been shown in mice to be essential for Ig class switching, recombination events that are required for the production of the various classes of secreted antibodies (33). Stat 6 contributes to class switching to produce IgE and IgG1 (11-13). In mice, IFN- $\gamma$, which acts via Stat 1 to activate the transcription of many early response genes, inhibits the transcription of germline I $\varepsilon$ and $\mathrm{I} \gamma 1$ and thereby blocks B cell production of IgE and IgG1 (34). This regulation appears to be mediated by the suppressor of cytokine signaling-1 (Socs-1), one of a new family of inhibitory Socs molecules defined by the presence of conserved SH2 domains and a novel motif termed a "Socs box" (35). These proteins are induced by cytokines and appear to function in a negative feedback loop. Socs-1 can bind to all the Jaks and inhibit Stat activation. The ability of IFN- $\gamma$ to inhibit IL- 4 signaling seems to be due to the induction of Socs- 1 and the resulting suppression of Stat 6 activity $(36,37)$.

A second inhibitor of Jak-Stat signaling implicated in atopic immune responses is Bcl-6, the product of a putative protooncogene that is rearranged or mutated in non-Hodgkin lymphomas. Normally, Bcl-6 functions as a transcriptional repressor, and it has been shown to bind to Stat6-binding sites, such as one found in the $I \varepsilon$ promoter. Mice lacking Bcl- 6 develop an inflammatory disease characterized by increased levels of IgE, Th2 cells, and mast cell infiltrates. Their B cells produce high levels of $\operatorname{IgE}(38,39)$, a phenotype that requires Stat 6 expression by these cells $(40,41)$. The importance of these regulators of Jak-Stat signaling in human asthma is still undefined.

\section{Stat6 in asthma pathophysiology}

In light of the extensive evidence that JAK-STAT signaling controls many of the physiologic events that are deregulated in asthma, several groups have pursued the role of this pathway in murine models of pulmonary inflammation and AHR. Most such studies have focused on Stat 6 , given its involvement in directing Th2 responses and IgE production (42-46). Following allergen provocation Stat 6 deficient-mice fail to develop a pulmonary Th2 response or AHR, and they exhibit no detectable increase in IgE production or in the number of mucus-containing cells. Because intravenous administration of IL-5 to Stat6-deficient mice restores the development of eosinophilia and AHR after antigen sensitization (46), it appears that one essential role of Stat 6 in the development of AHR is to drive Th2 responses and IL-5 production. Interestingly, different groups have reported distinct effects of Stat 6 deficiency on the inflammatory infiltrate. Mice of the BALB/c background show only a $50 \%$ decrease in the eosinophilic infiltrate, whereas antigen-induced eosinophilia is completely blocked in Stat6-deficient C57BL/ 6 mice $(42,43)$. Whether distinctions in sensitization protocols and/or strain background contribute to the differences reported remains to be established.

Recent reports suggest that the development of allergic pulmonary inflammation requires the activation of Stat 6 not only in T cells, but also in the parenchymal cells of the lung (47). Indeed, adoptively transferred antigen-specific Th2 cells from $\mathrm{Stat}_{6}{ }^{++}$mice fail to mediate allergic inflammation in Stat6-deficient mice. These defects are believed to be due to impairments of these Th2 cells to traffic to the lung, possibly because of defects in the production of chemokines like eotaxin, which control recruitment of Th2 cells. Another feature of asthma that appears to require Stat 6 is mucus production: Stat $6^{-/}$mice do not demonstrate goblet cell hyperplasia and have decreased mucus secretion in the ovalbumin-asthma challenge asthma model (42, 43, 48). Again, transfer of antigen-specific Stat $6^{+/+} \mathrm{T}$ cells fails to complement this defect, thus implicating Stat 6 within the lung as a key mediator of mucus production (47). Similar findings in terms of AHR have also been reported. It will be interesting to determine whether cell type-specific transcription factors can influence the effects of Stat 6 within specific cell types, given that IL-13 has recently been shown to activate different gene profiles in distinct human airway cell types (49). Taken together, all these studies strongly support the notion that Stat 6 plays a central role in the pathogenesis of asthma.

Several groups have also investigated the expression and activation of STAT6 in asthmatic individuals. Peripheral blood lymphocytes from asthmatic and allergic patients do not display significant differences in the level of STAT6 activity relative to healthy controls (50), but these patients do have a higher density of STAT6-expressing cells in their airways (51). Intriguingly, the density of these STAT6-expressing cells is significantly higher in atopic than in nonatopic asthmatics, although there is no significant difference in expression of the other characteristic Th2 transcription factors GATA3 and c-maf between these groups. One recent study of subjects with severe asthma confirms that such patients show significantly elevated airway levels of STAT6 and also identifies the 
major STAT6-expressing cell type in this tissue as the bronchial epithelial cell (52). Hence, the microenvironment of asthmatic airways may contribute to deregulating STAT6 expression. Further studies will be needed to investigate the functional consequences of the enhanced expression of STAT6 in asthmatic patients and to determine whether deregulated Stat6 expression is indeed a critical distinction between atopic and nonatopic asthma.

1. Beasley, R., Crane, J., Lai, C.K.W., and Pearce, N. 2000. Prevalence and etiology of asthma. J. Allergy Clin. Immunol. 105:S466-S472.

2. Wills-Karp, M. 1999. Immunologic basis of antigen-induced airway hyperresponsiveness. Annu. Rev. Immunol. 17:255-281.

3. Darnell, J.E. 1997. STATs and gene regulation. Science. 277:1630-1635.

4. Seder, R., and Paul, W. 1994. Acquisition of lymphokine-producing phenotype by CD4+ T cells. Annu. Rev. Immunol. 12:635-673.

5. Cohn, L., and Ray, A. 2000. T-helper type 2 cell-directed therapy for asthma. Pharmacol. Ther. 88:187-196.

6. Rengarajan, J., Szabo, S.J., and Glimcher, L.H. 2000. Transcriptional regulation of Th1/Th2 polarization. Immunol. Today. 21:479-483.

7. Murphy, K.M., et al. 2000. Signaling and transcription in T helper development. Annu. Rev. Immunol. 18:451-494.

8. Kopf, M., et al. 1993. Disruption of the murine IL-4 gene blocks Th2 cytokine responses. Nature. 362:245-248.

9. Noben-Trauth, N., et al. 1997. An interleukin 4 (IL-4)-independent pathway for CD4+ T cell IL-4 production is revealed in IL-4 receptor-deficient mice. Proc. Natl. Acad. Sci. USA. 94:10838-10843.

10. Wurster, A.L., Tanaka, T., and Grusby, M.J. 2000. The biology of Stat4 and Stat6. Oncogene. 19:2577-2584.

11. Kaplan, M.H., Schindler, U., Smilery, S.T., and Grusby, M.J. 1996. Stat6 is required for mediating responses to IL-4 and for the development of Th2 cells. Immunity. 4:313-319.

12. Takeda, K., et al. 1996. Essential role of Stat6 in IL-4 signalling. Nature. 380:627-630.

13. Shimoda, K., et al. 1996. Lack of IL-4-induced Th2 response and IgE class switching in mice with disrupted Stat6 gene. Nature. 380:630-633.

14. Finkelman, F.D., et al. 2000. Stat 6 regulation of in vivo IL-4 responses. J. Immunol. 164:2303-2310.

15. Grogan, J.L., et al. 2001. Early transcription and silencing of cytokine genes underlie polarization of $\mathrm{T}$ helper cell subsets. Immunity. 14:205-215

16. Kurata, H., Lee, H.J., O'Garra, A., and Arai, N. 1999. Ectopic expression of activated Stat 6 induces the expression of Th2-specific cytokines and transcription factors in developing Th1 cells. Immunity. 11:677-688.

17. Zhu, J., Guo, L., Watson, C.J., Hu-Li, J., and Paul, W.E. 2001. Stat6 is necessary and sufficient for IL-4's role in Th2 differentiation and cell expansion. J. Immunol. 166:7276-7281.

18. Zhang, S., Lukacs, N.W., Lawless, V.A., Kunkel, S.L., and Kaplan, M.H. 2000. Differential expression of chemokines in Th1 and Th2 cells is dependent on Stat6 but not on Stat4. J. Immunol. 165:10-14.

19. Kaplan, M.H., Daniel, C., Schindler, U., and Grusby, M.J. 1998. Stat proteins control lymphocyte proliferation by regulating p27Kip1 expression. Mol. Cell. Biol. 18:1996-2003.

20. Kaplan, M.H., Sun, Y.L., Hoey, T., and Grusby, M.J. 1996. Impaired IL-12 responses and enhanced development of Th2 cells in Stat4-deficient mice. Nature. 382:174-177.

21. Curiel, R.E., et al. 1997. Identification of a Stat6-responsive element in the promoter of the human interleukin-4 gene. Eur. J. Immunol. 27:1982-1987.

22. Georas, S.N., et al. 1998. Stat6 inhibits human interleukin-4 promoter activity in T cells. Blood. 92:4529-4538.

23. Kubo, M., et al. 1997. T-cell subset-specific expression of the IL-4 gene is regulated by a silencer element and STAT6. EMBO J. 16:4007-4020.

24. Thierfelder, W.E., et al. 1996. Requirement for Stat4 in interleukin-12 mediated responses of natural killer and T cells. Nature. 382:171-174.

25. Mullen, A.C., et al. 2001. Role of T-bet in commitment of TH1 cells before IL-12-dependent selection. Science. 292:1907-1910.

26. Szabo, S.J., et al. 2000. A novel transcription factor, T-bet, directs Th1 lineage commitment. Cell. 100:655-669.

27. Lighvani, A.A., et al. 2001. T-bet is rapidly induced by interferon-gamma in lymphoid and myeloid cells. Proc. Natl. Acad. Sci. USA. 98:15137-15142.
28. Finotto, S., et al. 2002. Development of spontaneous changes consistent with human asthma in mice lacking T-bet. Science. 295:336-338.

29. Xu, X., Sun, Y.L., and Hoey, T. 1996. Cooperative DNA binding and sequence-selective recognition conferred by the STAT amino-terminal domain. Science. 273:794-797.

30. Ouyang, W., et al. 1998. Inhibition of Th1 development mediated by GATA-3 through an IL-4-independent mechanism. Immunity. 9:745-755.

31. Milgrom, H., et al. 1999. Treatment of allergic asthma with monoclonal anti-IgE antibody. rhuMAb-E25 Study Group. N. Engl. J. Med. 341:1966-1973.

32. Jiang, H., Harris, M.B., and Rothman, P. 2000. IL-4/IL-13 signaling beyond JAK/STAT. J. Allergy Clin. Immunol. 105:1063-1070.

33. Coffman, R.L., Lebman, D.A., and Rothman, P. 1993. Mechanism and regulation of immunoglobulin isotype switching. Adv. Immunol. 54:229-270.

34. Linehan, L.A., Warren, W.D., Thompson, P.A., Grusby, M.J., and Berton, M.T. 1998. STAT6 is required for IL-4-induced germline Ig gene transcription and switch recombination. J. Immunol. 161:302-310.

35. Chen, X.P., Losman, J.A., and Rothman, P. 2000. SOCS proteins, regulators of intracellular signaling. Immunity. 13:287-290.

36. Venkataraman, C., Leung, S., Salvekar, A., Mano, H., and Schindler, U. 1999. Repression of IL-4-induced gene expression by IFN-gamma requires Stat 1 activation. J. Immunol. 162:4053-4061.

37. Dickensheets, H.L., and Donnelly, R.P. 1999. Inhibition of IL-4-inducible gene expression in human monocytes by type I and type II interferons. J. Leukoc. Biol. 65:307-312.

38. Dent, A.L., Shaffer, A.L., Yu, X., Allman, D., and Staudt, L.M. 1997. Control of inflammation, cytokine expression, and germinal center formation by BCL-6. Science. 276:589-592.

39. Ye, B., et al. 1997. The BCL- 6 proto-oncogene controls germinal-centre formation and Th2-type inflammation. Nat. Genet. 16:161-170.

40. Harris, M.B., et al. 1999. Transcriptional repression of Stat6-dependent interleukin-4-induced genes by BCL-6: specific regulation of iepsilon transcription and immunoglobulin E switching. Mol. Cell. Biol. 19:7264-7275.

41. Dent, A.L., Doherty, T.M., Paul, W.E., Sher, A., and Staudt, L.M. 1999. BCL-6-deficient mice reveal an IL-4-independent, STAT6-dependent pathway that controls susceptibility to infection by Leishmania major. J. Immunol. 163:2098-2103.

42. Kuperman, D., Schofield, B., Wills-Karp, M., and Grusby, M.J. 1998. Signal transducer and activator of transcription factor 6 (Stat6)-deficient mice are protected from antigen-induced airway hyperresponsiveness and mucus production. J. Exp. Med. 187:939-948.

43. Akimoto, T., et al. 1998. Abrogation of bronchial eosinophilic inflammation and airway hyperreactivity in signal transducers and activators of transcription (STAT)6-deficient mice. J. Exp. Med. 187:1537-1542.

44. Miyata, S., et al. 1999. STAT6 deficiency in a mouse model of allergeninduced airways inflammation abolishes eosinophilia but induces infiltration of CD8+ T cells. Clin. Exp. Allergy. 29:114-123.

45. Herrick, C.A., MacLeod, H., Glusac, E., Tigelaar, R.E., and Bottomly, K. 2000. Th2 responses induced by epicutaneous or inhalational protein exposure are differentially dependent on IL-4. J. Clin. Invest. 105:765-775.

46. Tomkinson, A., et al. 1999. The failure of STAT6-deficient mice to develop eosinophilia and airway hyperresponsiveness is overcome by interleukin-5. Am. J. Respir. Crit. Care Med. 160:1283-1291.

47. Mathew, A., et al. 2001. Signal transducer and activator of transcription 6 controls chemokine production and T helper cell type 2 cell trafficking in allergic pulmonary inflammation. J. Exp. Med. 193:1087-1096.

48. Tomkinson, A., et al. 2001. Temporal association between airway hyperresponsiveness and airway eosinophilia in ovalbumin-sensitized mice. Am. J. Respir. Crit. Care Med. 163:721-730.

49. Lee, J.H., et al. 2001. Interleukin-13 induces dramatically different transcriptional programs in three human airway cell types. Am. J. Respir. Cell Mol. Biol. 25:474-485.

50. Miller, R.L., Eppinger, T.M., McConnell, D., Cunningham-Rundles, C., and Rothman, P. 1998. Analysis of cytokine signaling in patients with extrinsic asthma and hyperimmunoglobulin E. J. Allergy Clin. Immunol. 102:503-511.

51. Christodoulopoulos, P., et al. 2001. Th2 cytokine-associated transcription factors in atopic and nonatopic asthma: evidence for differential signal transducer and activator of transcription 6 expression. J. Allergy Clin. Immunol. 107:586-591.

52. Mullings, R.E., et al. 2001. Signal transducer and activator of transcription 6 (STAT-6) expression and function in asthmatic bronchial epithelium. J. Allergy Clin. Immunol. 108:832-838. 